\title{
Perils of dermal fillers
}

\author{
S. D. Colbert, ${ }^{1}$ B. J. Southorn, ${ }^{* 2}$ P. A. Brennan ${ }^{2}$ and V. Ilankovan ${ }^{1}$
}
IN BRIEF
- Raises awareness among practitioners of a long-term complication that occurred following use of cosmetic dermal filler.
- Reports on the histopathology of lesions concerned and the pathological process involved.
- Highlights the implications surrounding informed consent and the importance of being aware of potential risks when advising patients.

\begin{abstract}
With the increasing use of hyaluronic acid-based injectable fillers for cosmetic enhancement a variety of adverse reactions are being reported in the literature. Although most adverse outcomes occur early we describe an interesting case study of a female presenting with granulomatous complications ten years postoperatively. To our knowledge this is one of the longest reported delayed reactions. For the general dental practitioner offering such treatments it is important to be fully aware of the potential risks and how they may be managed in order to consent appropriately for cosmetic procedures.
\end{abstract}

\section{INTRODUCTION}

Using injectable fillers is one of the most popular treatment options for managing the ageing face. In recent years, the rate of soft tissue augmentation has exponentially increased for facial rejuvenation. It has become a particularly attractive option for patients because the results are easy to appreciate immediately postinjection and have a predictable longevity. General dental practitioners (GDPs) with a special interest in aesthetics have used injectable fillers because, with proper patient selection and injection technique, most dermal fillers are able to modify the appearance/fullness of the skin and soft tissues whilst offering an impressive safety profile. There are many different fillers available for augmentation and correction of soft tissue deficiencies, but there is risk associated with their use. Adverse reactions including erythema, papules or nodules and abscesses may appear and usually occur early. Although fillers are generally regarded as safe, unanticipated events and adverse outcomes can occur with these agents. To

Poole General Hospital NHS Trust, Department of Oral and Maxillofacial Surgery, Longfleet Road, Poole, BH15 2JB; ${ }^{2}$ Queen Alexandra Hospital, Maxillofacial Surgery, QAH Portsmouth, Cosham, Portsmouth, P06 3LY

*Correspondence to: Miss Bryony Southorn Email: bryonysouthorn@hotmail.co.uk

\section{Refereed Paper}

Accepted 22 January 2013

DOI: 10.1038/sj.bdj.2013.326

${ }^{\bullet}$ British Dental Journal 2013; 214: 339-340 prevent complications and treat potential issues appropriately, it is of the utmost importance that GDPs fully understand the perils of dermal fillers.

We present an unusual extensive granulomatous reaction occurring ten years after an injection of a temporary/semipermanent filler.

\section{CASE REPORT}

A 65-year-old female had perioral augmentation with the injectable dermal filler DermaLive ${ }^{\circledR}$ (Dermatech, Intradermal Distribution Inc) in the nasiolabial folds. Ten years later she presented with a severalyear history of a progressively increasing number of hard lumps bilaterally in areas that had been injected with DermaLive ${ }^{\circledR}$ (Fig. 1). A biopsy showed florid granulomatous inflammation in the dermis and subcutis with many multinucleate giant cells. There were multiple pink, translucent, extracellular and non-birefringent fragments within cystic spaces consistent with a foreign body granulomatous reaction (Fig. 2). The filler component that induced this reaction was the methylmethacrylate.

\section{DISCUSSION:}

Cross linking of molecules is a recognised manufacturing technique to prolong the duration and effect of temporary fillers. DermaLive $^{\circledR}$ is a synthetic, semi-permanent, non-biodegradable injectable dermal filler that lasts an undefined but prolonged time in the dermis. It is composed of $60 \%$

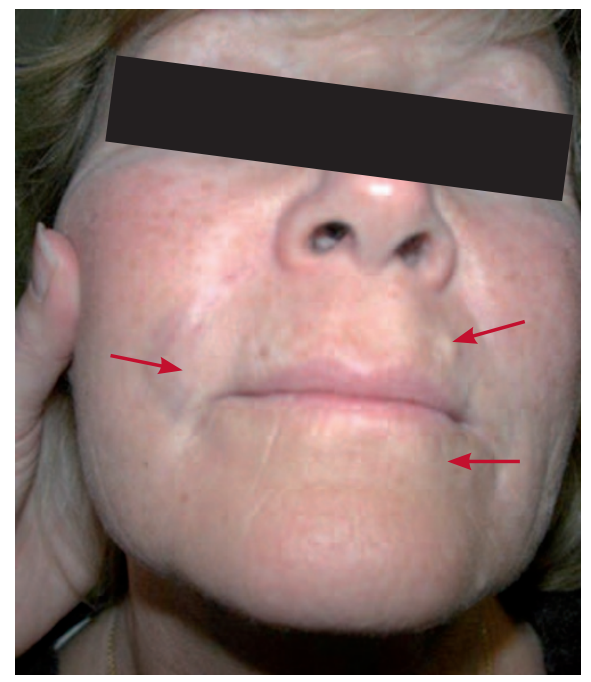

Fig. 1 Increasing number of hard lumps in the areas injected with Dermalive ten years earlier (indicated by arrows)

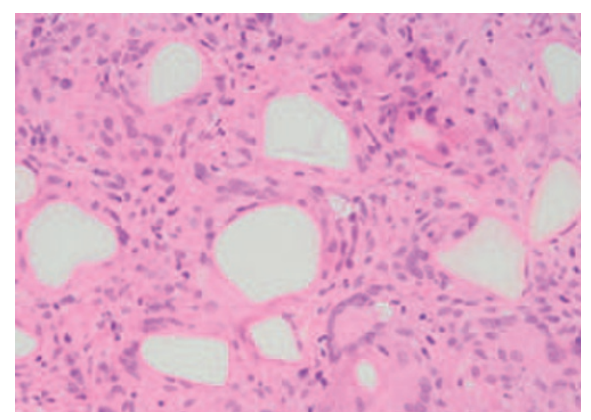

Fig. 2 Granulomatous inflammation with sharply circumscribed cystic spaces containing filler component within the multinucleated giant cells (haematoxylin-eosin; original magnification $\times 40$ )

crosslinked hyaluronic acid gel vector 40\% acrylic hydrogel particles.

The incidence of developing granulomatous reactions has been reported as less 
than 1.2 per 1,000 , occurring on average six months after injection. These unusual reactions are difficult to manage and can appear months to years after initial exposure. ${ }^{1}$ The longest reported time period for developing a granulomatous reaction is 48 months. $^{2}$

The underlying aetiology for a granulomatous reaction to DermaLive ${ }^{\circledR}$ is unknown. Factors influencing their development include properties of the filler, the volume injected and previous infection or trauma, hypersensitivity reaction to the hyaluronic acid carrier or an underlying or associated infection. ${ }^{3}$ Tyssen and Menne suggest that an allergic contact dermatitis response to the methylmethacrylate monomers may be an important aetiological factor. ${ }^{4}$ The shape of the microspheres may induce a more severe granulomatous reaction. ${ }^{5}$

The formation of sterile abscesses raises questions regarding what triggered the inflammatory response. Activation of the immune system would involve an interaction between an immunogenic protein and the host immune cells. Inappropriate inflammatory responses, such as hypersensitivity reactions, also occur in response to invading proteins. As a glycosaminoglycan, hyaluronic acid is not a protein and accordingly, allergic reactions are rare. ${ }^{6}$ It can only be speculated as to the cause of the inflammatory response observed in our case.
Several treatment options exist. All are more experience-based than evidence-based and include local steroid injection and laser excision, but both have limitations. ${ }^{7}$

To our knowledge this case is possibly one of the longest reported delayed reactions to dermal fillers and with permanently scarred soft tissues. In a litigious society general dental practitioners using these fillers should be aware of these possible complications.

As with any cosmetic procedure, receiving informed consent and having effective communication with the patient before treatment is paramount. The increasing use of non-surgical aesthetic procedures in the general practice setting (thereby making them more available to the public) may lead to more cases of adverse reactions to dermal fillers. Once granulomas form in perioral areas it is not an easy problem to solve. Public and professional education is essential to avoid such perils.

\section{CONCLUSION}

Soft tissue augmentation with filler agents is in high demand, largely due to increased public exposure to these products and increasing confidence that these agents provide a safe and consistent means of facial rejuvenation. Despite the impressive safety profile of these products, complications do occur. It is important for the GDP to be knowledgeable about the perils of dermal fillers and select the product most likely to address the patient's concerns. If a granulomatous reaction does occur, thorough understanding of the diagnosis and treatment algorithms will help the injector safely navigate through this circumstance to minimise long-term sequelae. This article helps the GDP understand fillers and their complications in a way that will allow them to successfully avoid, accurately diagnose and efficiently manage potential granulomatous adverse events.

1. Vargas-Machuca I, Gonza'lez-Guerra E, Angulo J et al. Facial granulomas secondary to DermaLive microimplants: report of a case with histopathologic differential diagnosis among the granulomas secondary to different injectable permanent filler materials. Am J Dermatopathol 2006; 28: 173-177.

2. Alijotas-Reig J, Garcia-Gimenez V. Delayed immune-mediated adverse effects related to hyaluronic acid and acrylic hydrogel dermal fillers: clinical findings, long-term follow-up and review of the literature. J Eur Acad Dermatol Venereol 2007; $1-12$.

3. Maas C S, Papel I D, Greene D et al. Complications of injectable synthetic polymers in facial augmentation. Dermatol Surg 1997; 23: 871-877.

4. Tyssen J P, Menne' T. Allergic reaction to hydroxyethyl-methacrylate following intradermal filler injection. Contact Dermatitis 2005; 52: 341-342.

5. De Boulle K. Management of complications after implantation of fillers. J Cosmet Dermatol 2005; 3: $2-15$.

6. Andre P, Flechet M L. Angioedema after ovine hyaluronidase injection for treating hyaluronic acid overcorrection. J Cosmet Dermatol 2008: 7: $136-138$

7. Angus J E, Affleck A G, Leach I H, Millard L G. Two cases of delayed granulomatous reactions to the cosmetic filler DermaLive, a hyaluronic acid and acrylic hydrogel. Br J Dermatol 2006; 154: $1077-1078$

\section{Corrigendum}

\section{Practice articles (BDJ 2012; 214: 155-158, 227-231)}

'An overview of electronic apex locators: part 1' and 'An overview of electronic apex locators: part 2'

In the above practice articles, the resistance given in Figure 4 (BDJ 2012; 214: 155-158) and Figure 1 (BDJ 2012; 214: 227-231) should have read $6.5 \mathrm{k} \Omega$. Similarly, the following sentence (BDJ 2012; 214: 227) is incorrect:

'the electrical resistance between an endodontic instrument at the apical foramen and an electrode attached to oral mucous membrane was approximately $6.5 \mathrm{~kW}$ :

This should have read 'approximately $6.5 \mathrm{k} \Omega$ '

The authors apologise for any confusion caused. 